\title{
Characterizing Eating Disorder Issues on Sina Weibo
}

\author{
Jingyun Tang, Guang $\mathrm{Yu}^{1}$, Zheng Wang, Xiaoxu Yao \\ School of Management, Harbin Institute of Technology, Harbin, Heilongjiang, China
}

\begin{abstract}
Eating disorder (ED) was not learned well in China. To contribute to an understanding of the prevalence of EDs in China and to identify individuals with ED tendencies, we randomly selected one million Sina Weibo (social media platform similar to twitter) users and collected all their postings related to ED keywords. Using thematic analysis and sentiment analysis our study proposes an approach for identifying ED-prone people, the use of which reveals the current situation with regard to EDs in China. The identified ED-prone people were all willing to share information about diet, body shape and slimming while reporting their disease condition. Most of these users were young females, who were active on social media but in a negative frame of mind. With the method proposed in this study, it is possible to identify individuals with an ED and carry out timely intervention via social media at a very low cost.
\end{abstract}

Keywords. Eating disorder; anorexia; social media; Sina Weibo

\section{Introduction}

The mantra of "regard being slender as beauty" has come to influence the perception of beauty among Chinese in recent years, and extreme measures taken to lose weight have led to eating disorders (EDs) that have become a serious problem hindering the healthy development of Chinese adolescents $[1,2]$. Presently about $0.5 \%$ to $2 \%$ of people worldwide, dominated by teenagers, are suffering from an eating disorder (ED)[3], which includes incomplete forms of anorexia nervosa and bulimia nervosa. With a fatality rate of $5 \%$ to $6 \%$, anorexia nervosa (AN) is the most fatal of all mental diseases[4].

Since individuals usually try to conceal ED symptoms[5]. It is particularly difficult to detect people with an ED in order to treat them in a timely manner. Given the often secretive nature of EDs, those with an ED may seek social support or resources online. Online social media offers anonymity and thus users have few scruples in expressing themselves, which makes social media the best tool for those with an ED to search for other users suffering from the same disease whom they can discuss their problems with, and seek support and advice from[6, 7]. The emergence of social media platforms provides an effective method to identify individuals with an ED and to help understand the development of EDs at a very low cost.

Sina Weibo, which is similar to Twitter, is the largest platform of user information sharing, circulation and acquisition in China. In the past two years, one of the hot

\footnotetext{
${ }^{1}$ Corresponding Author: Guang Yu, Harbin Institute of Technology, No.92, Xidazhi Street, Nangang District, Harbin City, Heilongjiang Province, China; Email:yug@hit.edu.cn
} 
topics on Weibo has been the "skinny craze". For example, the posts of "A4 waist" (the width of the waist is smaller than that of an A4 paper, namely, 210mm) has reached 270 million and the number of people participating in the discussion has reached 302,000. Another example is the throwing down of "skinny craze" challenges by posting photos. These kinds of activities are undoubtedly putting potential ED people at risk. In addition, because of the openness and anonymity of Weibo, users may post and discuss private matters without scruples. Therefore, it is worthwhile to probe into China's ED population using Weibo data sources.

\section{Literature Review}

In recent years, more and more researchers use social media data to study psychological diseases[8,9]. The research on ED based on social media mainly focuses on the following aspects:

First, the relationship between the social media and ED has been mainly explored. Some researchers have found that the frequent use of social media had a stronger correlation with body image concerns and ED[10-12]. A study showed positive linear relationship between the time of social media use and reported ED behaviors[13,14]. In addition, a study showed that there is a link between online ED content browsing and offline ED behavior[15].

Second, some studies focus on online Pro-ED community[16]. Some studies by the hashtag (for example, \#proana, pro-ED) or keywords found those communities openly supporting ED on social media[17]. Through content analysis, it was found that the community content was consistent with the behavior of ED psychiatry, and the community members expressed support for these behaviors[18]. After the characteristics and posting information of users in these communities were tracked and analyzed, it was found that all such communities aggravate ED[19]. A study have established text classifiers to identify the risk pro-ED information by characterizing removed Pro-ED social media content[20].

Finally, studies from another perspective have also found that the Internet and social media can act as a platform to provide ED assessment and intervening measures, to decrease body image dissatisfaction and disordered eating patterns, and to reduce the morbidity of high-risk groups[21, 22]. The Internet and social media can also reduce the barriers for treatment, and provide an easy anonymous environment to encourage ED groups to conduct self-management[23, 24].

Therefore, social media does not only intensify ED hazards but is also the best methods for medical personnel to conduct assessments and take intervening measures. However, most of the existing research is based on Facebook and twitter, which are not available in China. As the prevalence of eating disorders in China is increasing year by year, it is necessary to investigate ED in social media.

In this study, an investigation is carried out into the population with EDs in China by extracting data from Weibo. Firstly, a general description of the current situation of EDs in China and an analysis of ED topics that users discuss via social media is provided; secondly, the personal features of ED users in China are revealed. Findings from this study are expected to provide a theoretical basis for the active intervention and treatment of ED users via social media. 


\section{Methods}

\subsection{Data collection}

API (Application Programming Interface) was used to randomly collect approximately 394 million postings of one million users from Weibo. These users were randomly sampled and all their postings were collected. These original data included posting text, posting time, and user information such as age, gender, region, number of followings, and number of followers. These data were publicly available but the study hides all traceable user information for the protection of the users' privacy. Ethical review was not required because we only used publicly available data in this study.

Selection of postings

\subsection{Selection of postings}

From the data sets collected, we retrieved all posting texts related to ED by keyword search. This was achieved by reading a plethora of articles and postings on Chinese ED websites and post bars in order to find words that best embody the characteristics of ED. To avoid collecting noisy data, some commonly used words and terms such as dieting, slimming, and losing weight were not adopted. All postings that contained the keyword "eating disorder ", "anorexia", "bulimia", "emetic", "self-induced vomiting", were retrieved from the postings. The number of the selected ED-related postings was 33,230 .

Not all posts containing the keyword are disclosed ED. In order to extract the content related to ED, two trained researchers with mental health experience then independently labeled the postings and a codebook was created for differentiating the 33,230 postings according to content; they coded all the postings by using the codebook independently[25]. Each of the postings was discussed and an agreement on the final assigned code was made. A third $\mathrm{PhD}$ researcher was introduced to code a sample of 1,000 postings randomly selected from all 33,230 postings to compute the inter-rater reliability. Cohen's kappa test is often used to check the inter-coder reliability[26,27]. The inter-rater reliability for each theme was as follows.

A. Users suffering from ED (kappa $>0.4)$

B. Users express wish to develop AN (kappa $>0.4$ )

C. Users disclosing the behaviors of disordered eating $($ Kappa $<0.4)$

D. Sharing medical information on ED, news, exaggeration (Kappa $>0.4$ )

Obviously, not all postings that contained keyword(s) reflect disclosure of ED(type D), and the quantity and kappa value for disclosing the behaviors of disordered eating were too small(type C). Finally, 1,145 postings (type A) and 96 postings (type B) were identified as disclosure of an ED and adopted for further analysis.

\subsection{Themes}

To identify ultimate themes of the postings that expressed disclosure of an ED, the two experienced members separately coded each of the 1,145 (type A) postings and 96 (type B) postings. The third member then was introduced to code the postings. Finally, Cohen's kappa test was conducted. 


\subsection{Demographics characteristics}

It should be noted that 1,114 users posted the 1,145 postings that expressed symptoms of suffering from ED, while 94 users posted the 96 postings that expressed a wish to develop AN - these two types of users are referred to as eating disorder-prone (ED-prone) users. To verify the result, 910,000 users were randomly selected from Weibo as a comparison group, and Pearson chi-square was adopted to compare the characteristics of ED-prone users and typical users (from the comparison group) on the gender distribution. Wilcoxon Rank Sum and Signed Rank Test[28] were used to determine whether the medians of user's age, postings, followings and followers were statistically significant between the ED and typical users. $\mathrm{P}<0.05$ was considered statistically significant.

\subsection{Sentiment analysis}

To explore the emotional states of the postings posted by ED-prone users, all of their postings were retrieved from the database. The number of these postings was 775,066 and 61,930. For comparison, 120,000 postings from typical users were randomly selected.

It is impossible to classify a large number of postings manually, so we need to build a text classifier. A long short-term memory(LTSM) network is a type of recurrent neural network (RNN)[29], which is widely used in natural language processing tasks. Based on the LTSM algorithm, sentiment classifiers were built. Detection was considered as a binary classification problem (emotion states of each posting, namely, positive or negative). For the supervised classification problem, a training data set is necessary. Therefore, 30,000 postings were randomly selected from the postings of 1 million users. Three trained researchers independently labeled the postings, and majority rule was adopted when there was disagreement. Ten-folded cross-validation and accuracy were selected as measures to assess the performance of the classifiers. Experimental results suggested that the classifiers achieved the highest accuracy with $93 \%$.

After the emotional state of each post was determined, the negative emotion score of each user was calculated; that is, the proportion of the number of postings with negative emotion stated to that of the total postings of a user. The higher the proportion is, the stronger the user's negative emotion.

\subsection{Influencing factors of negative emotion and mental health}

To identify the influencing factors of negative emotion of ED-prone users, the 1,000 posts randomly selected from all acquired postings with negative emotion states, were labeled. Users having a high negative emotion score were also examined to see whether they posted other psychological comorbidity messages. 


\section{Results}

\subsection{Themes}

Postings containing the keywords were selected. The majority of postings were excluded because of sharing medical information and because kappa values were too small. Finally, 1,145 postings (type A) were identified as users suffering from an ED, in which AN accounted for $89.69 \%$ and bulimia $8.47 \%$. In addition, 96 postings (type B) expressed their wish to develop AN. These postings were further coded to explore the themes of EDs in the postings. Themes identified in the postings are presented in Table 1.

Table 1 Themes of ED-related postings.

\begin{tabular}{|c|c|c|c|c|}
\hline & Thematic analysis & $\begin{array}{l}\text { Kappa } \\
\text { value }\end{array}$ & $\begin{array}{l}\text { Quantity } \\
\text { (proportion) }\end{array}$ & example \\
\hline \multicolumn{5}{|c|}{ Type A } \\
\hline 1 & $\begin{array}{l}\text { disclosure of ED } \\
\text { only }\end{array}$ & 0.880 & $423,36.94 \%$ & $\begin{array}{l}\text { I am tormented by AN, and will die soon! This is } \\
\text { God's punishment to me, and I will take the } \\
\text { consequences! }\end{array}$ \\
\hline 2 & $\begin{array}{l}\text { description of } \\
\text { symptoms and } \\
\text { behaviors, } \\
\text { including eating, } \\
\text { body shape, and } \\
\text { slimming }\end{array}$ & 0.833 & $610,53.28 \%$ & $\begin{array}{l}\text { I still fail to do exercises but be tormented by } \\
\text { Bulimia today./Now I am sitting in the classroom, } \\
\text { and only thinking about which snack to eat./After } \\
\text { calculation, I found the snack's calorific value is } \\
\text { more than } 1,000 \text { kilocalories /If I eat the snack, at } \\
\text { least two days of my exercises will lose effect. }\end{array}$ \\
\hline 3 & seeking help & 0.614 & $74,6.46 \%$ & I have an eating disorder, who can help me? \\
\hline 4 & taken measures & 0.556 & $38,3.31 \%$ & I must have a good meal to overcome ED. \\
\hline 1 & $\begin{array}{l}\text { Type B } \\
\text { expressing wish to } \\
\text { develop AN only }\end{array}$ & 0.780 & $88,91.67 \%$ & I hope to develop anorexia. \\
\hline 2 & $\begin{array}{l}\text { seeking a method } \\
\text { for develop AN }\end{array}$ & 0.780 & $8,8.25 \%$ & I want to develop anorexia, and who can help me? \\
\hline
\end{tabular}

For type A, the most common theme of the postings $(n=610,53.28 \%)$ was describing their condition and behavior in eating, body shape, slimming and so on. Most users take pleasure in sharing and exchanging their own ED condition. The second most popular theme was disclosure of ED $(423,36.94 \%)$. Only $6.46 \%(n=74)$ of the postings attempted to seek help from others. About $3 \%(n=38)$ of the postings mentioned some measures taken to prevent or reduce ED. For type B, the most common theme of the postings $(n=88,91.67 \%)$ was expressing a wish to develop AN only.

\subsection{Demographic characteristics}

To better understand the difference between ED-prone users and typical users, it was decided to character the characteristics of the ED-prone Weibo users. Five variables of gender, age, number of postings, number of followings, and number of followers were used. 
Table 2 Demographic characteristics of ED-prone users

\begin{tabular}{|c|c|c|c|c|c|}
\hline & typical users & $\begin{array}{l}\text { A Users suffering } \\
\text { from ED }\end{array}$ & $\mathbf{P}_{\boldsymbol{A}}$ & $\begin{array}{l}\text { B Users express } \\
\text { wish to develop AN }\end{array}$ & $\mathbf{P}_{B}$ \\
\hline \multicolumn{6}{|l|}{ Gender } \\
\hline Male & $417180(43.79 \%)$ & $196(17.59 \%)$ & $\mathrm{P}<0.001$ & $9(9.57 \%)$ & $\mathrm{P}<0.001$ \\
\hline Female & $535577(56.21 \%)$ & $918(78.19 \%)$ & & $85(90.43 \%)$ & \\
\hline Age & $(10-60)$ & $(15-38)$ & & $(17-33)$ & \\
\hline $25 \%$ & 21 & 22 & & 20 & \\
\hline Median & 24 & 24 & $\mathrm{P}=0.01443$ & 23 & $\mathrm{P}=0.01524$ \\
\hline $75 \%$ & 28 & 26 & & 26 & \\
\hline \multicolumn{6}{|c|}{$\begin{array}{l}\text { Number of } \\
\text { postings }\end{array}$} \\
\hline $25 \%$ & 96 & 318 & & 247 & \\
\hline Median & 280 & 686 & $\mathrm{P}<0.001$ & 485 & $\mathrm{P}<0.001$ \\
\hline $75 \%$ & 728 & 1311 & & 1133 & \\
\hline \multicolumn{6}{|c|}{$\begin{array}{l}\text { Number of } \\
\text { followings }\end{array}$} \\
\hline $25 \%$ & 92 & 140 & & 138 & \\
\hline Median & 185 & 242 & $\mathrm{P}<0.001$ & 277 & $\mathrm{P}<0.001$ \\
\hline $75 \%$ & 341 & 421 & & 454 & \\
\hline \multicolumn{6}{|c|}{$\begin{array}{l}\text { Number of } \\
\text { followers }\end{array}$} \\
\hline $25 \%$ & 67 & 147 & & 124 & \\
\hline Median & 141 & 246 & $\mathrm{P}<0.001$ & 228 & $\mathrm{P}<0.001$ \\
\hline $75 \%$ & 280 & 386 & & 472 & \\
\hline
\end{tabular}

Table 2 shows the comparative result between demographic characteristics of groups with ED-prone and typical groups. The result of Pearson's chi-squared test shows that the gender distribution difference between the two groups has statistical significance. Analysis of gender distribution illustrates that females tend to have an ED more frequently than males, and a higher proportion of female users express a wish to develop AN. Results of Wilcoxon Rank Sum and Signed Rank Test show a statistical significance in the median difference in users' age, number of postings, followings, and followers. The age distribution was relatively young. Both the ED-prone users are more active and both their number of postings and followings are larger than those of typical Weibo users.

\subsection{Sentiment analysis}

Through sentiment classification of all postings of ED-prone users, it was found that compared with typical users, ED-prone users show stronger negative emotion.

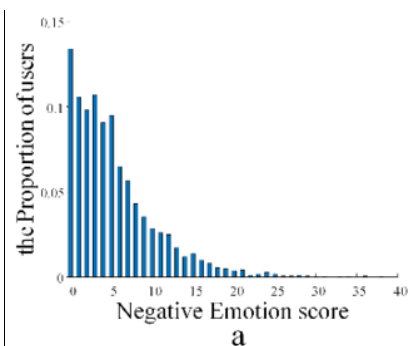

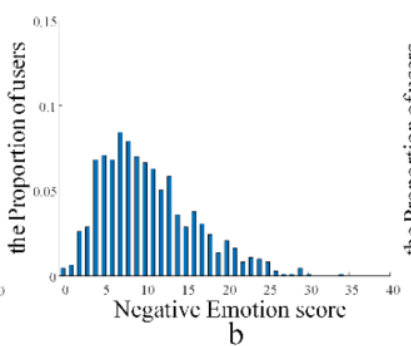

b

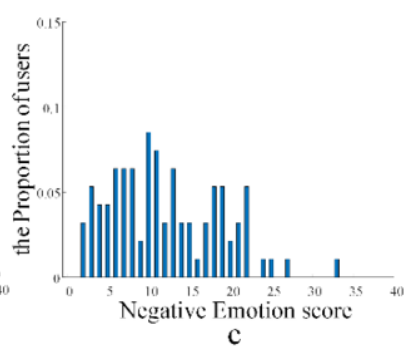

c

Figure 1 Distribution of negative emotion score of users; abscissa: negative emotion score, ordinate: the proportion of users 
A distribution graph of negative emotion score of each user was produced (see Figure 1). It can be seen that most of the typical users had a low negative emotion score (Figure 1(a)). In most cases however, among users suffering from an ED more negative emotion exist (Figure 1(b)); among the users expressing a wish to develop AN, there was generally deeper negative emotion and a relatively average distribution of scores (Figure 1(c)), which might be related to the limited data size.

\subsection{Influencing factors of negative emotion and mental health}

The main reasons for negative emotion reflected by the sampled posts and the corresponding proportions are as follows:

A. Physical complaint, such as illness, stomachache, hunger, insomnia, etc. (23\%)

B. Dissatisfied with self-image (4\%)

C. Life stress; work, study, and family affairs (33\%)

D. Weather and environment (7\%)

E. Not mentioned (33\%)

Users with high negative emotion score also disclosed other mental problems: 53 users posted that they were suffering from depression, 14 users posted that they had anxiety disorder, and 13 users out of these showed a suicidal tendency more than once.

\section{Discussion}

This study examined the postings in Weibo related to those who are ED-prone, which reflects the status of EDs in China and identifies the problems of managing ED-prone users.

From the sample database, it was found that most of the 1,114 users posted that they were suffering from the ED appeared to have AN. These 1,114 users accounted for a relatively small proportion of the selected users. In the data labelling process, a large part of the data was deleted because of some users' misunderstanding of AN. Most of them considered a poor appetite for a period of time as a sign of $\mathrm{AN}$, which indicates that there is a lack of general knowledge of EDs in China and that the majority are not aware of the AN disease.

The proportions of different themes of Weibo postings related to ED-prone users suggests that the users, in most cases, tend to talk about their own conditions because they might have no idea of how to seek help, or feel reluctant to ask for assistance with overcoming their ED. Those themes play an important role in establishing stronger links between these users and the exchange of information is expected to facilitate the identification of more online users with an ED. Therefore, it is necessary to proactively identify this group of users and render assistance by offering low-cost online intervention measures promptly to prevent the disease from getting worse.

In this study, 94 users expressed a wish to have AN. These users might be under great pressure imposed by the concept that regards being slender as beautiful, which has had a dramatic impact on the public through vigorous media promotions. Secondly, a group of pro-AN users (e.g., "Pro-Ana") is likely to emerge on the Internet and social media if there is no efficient control over relevant information and the users themselves. Studies have revealed that the users of "Pro-Ana" websites or online communities tend to share extreme weight loss measures with each other and learn skills via the Internet. In addition, these active and influential websites and online communities tend to be a risk factor for deterioration of EDs among users. To mitigate the risk, online guidance for correctly grasping healthcare concepts should be provided for the public and proper 
measures for weight control and beneficial healthcare should be introduced to reduce the incidence of EDs.

The study also statistically analyzed the demographic characteristics of ED-prone users. First, gender distribution is consistent with previous studies by finding many more female users than male users[11, 30]. This significant gender difference implies that more attention should be paid to female users in future studies so as to customize health information provided during online management and control. Second, from the age statistics we found that users between the ages of 20 to 30 years old account for the largest proportion among users who are ED-prone. Previous studies usually restricted samples to teenagers under 18 years old or college students[2, 31]. However, such sample selection is unsuitable for studies on EDs in China. Therefore future studies should enlarge the age range of samples. Third, the number of postings, followings, and followers of both the two types of users are much larger than those of typical users. We think this may be related to the lack of identity in 'real life' and so they take pleasure in exchanging information and acquiring support through the anonymous environment of social media. However, since it is easy for them to receive wrong health information this way, it is very important to carry out online management and control for ED-prone users.

This study found that most of the ED-prone users showed negative emotions and tended to complain about their poor health, such as stomachache or hunger that might result from an ED. There are potential factors brought on by such negative emotions, such as discontentment with oneself, tremendous pressure, and anxiety, all of which are likely to cause or aggravate EDs. Moreover, identification of these factors may be essential for the treatment of the disease. These findings about physical and mental phenomena enable professionals to provide online assistance and guidance for users in terms of psychotherapy, nutrition counseling, and foundation of support groups. Apart from this, it was also noted that those with strong negative emotions had other psychological comorbidities, such as depression, anxiety disorder and even suicidal tendencies. The results suggest that the effects and treatment of these comorbidities should be taken into consideration when prescribing treatment and developing prevention plans for patients suffering from an ED.

Without further study or diagnosis by professional doctors, we cannot be sure whether the users in the study reporting their ED are really developing an ED. However, we think these users are potential ED patients, and this is an important step for helping ED patients. In addition, some users in their postings do not specifically mention the term ED but instead describe their extreme slimming behavior. Furthermore, most postings in Weibo only include one or two sentences, which restrict demarcation of EDs and as a result such content failed to pass the Cohen's kappa test and were not analyzed. Further studies are needed for identifying the relationship between particular symptoms and user activity on social media.

All in all, EDs are a serious and even fatal disease. This paper is the first to carry out an investigation of EDs based on social media. Most of the previous studies identified potential ED patients through traditional epidemiology or clinical methods that are resource and time intense. Nevertheless, this study provides medical researchers and health care service organizations with a smart method at a lower cost to identify and efficiently assist the population with EDs. It allows professionals to offer healthcare information and psychological guidance online, provide ED-prone users with assistance to combat the disease in the earlier stages, and strengthen online regulations on risk factors for EDs; more studies should be conducted to facilitate the 
implementation of these plans. It is hoped that the findings of this study can enable mental health organizations in China to obtain a deeper understanding of the condition of the ED population and play a significant part in effectively preventing EDs and reducing incidence of the disease in China.

\section{Conclusion}

This is the first survey of ED on Chinese social media. We use keyword method to identify individuals with ED tendency. Through themes analysis, we find that ED-prone individuals are willing to share and exchange their own ED condition, and unwilling to seek help actively. Some users have made public their desire to get AN. These behaviors are very dangerous, which show there is still a lack of awareness of ED problem in China. According to the demographic characteristics, most of ED users are young women and more active than the general users. In addition, the sentiment analysis is conducted to estimate the negative emotion of ED-prone users of social media and the possibility of suffering from other mental comorbidities. It is possible that the findings of this study enable mental health organizations in China to obtain a deeper understanding of the condition of the ED population. This study also plays a significant role in effectively preventing EDs and reducing incidence of the disease in China.

\section{References}

[1] Lian, Q. G.; Zuo, X. Y.; Mao, Y. Y.; Luo, S.; Zhang, S. C.; Tu, X. W.; Lou, C. H.; Zhou, W. J. Anorexia nervosa, depression and suicidal thoughts among Chinese adolescents: a national school-based cross-sectional study. Environ Health Prev 2017, 22-30. 10.1186/s12199-017-0639-2.

[2] Chang, W.-w.; Nie, M.; Kang, Y.-w.; He, L.-p.; Jin, Y.-1.; Yao, Y.-s., Subclinical eating disorders in female medical students in Anhui, China: a cross-sectional study. Nutr. Hosp. 2015, 31,1771-1777. 10.3305/nh.2015.31.4.8456.

[3] Campbell, K.; Peebles, R., Eating disorders in children and adolescents: state of the art review. Pediatrics 2014, 134, 582-592. 10.1542/peds.2014-0194.

[4] Arcelus, J.; Mitchell, A. J.; Wales, J.; Nielsen, S., Mortality rates in patients with anorexia nervosa and other eating disorders: a meta-analysis of 36 studies. Arch Gen Psychiat. 2011, 68, 724-731. 10.1001/archgenpsychiatry.2011.74

[5] Richardson, R.; Richards, D. A., Designing a search strategy for locating self-help books for people with depression. Health Info Libr J 2006, 23, 294-295. 10.1111/j.1471-1842.2006.00667.x

[6] Brotsky, S. R.; Giles, D., Inside the "pro-ana" community: A covert online participant observation. Eat. Disord. 2007, 15, 93-109. 10.1080/10640260701190600

[7] Fox, N.; Ward, K.; O'rourke, A., Pro - anorexia, weight - loss drugs and the internet: an 'anti recovery'explanatory model of anorexia. Sociol. Health Ill. 2005, 27, 944-971. 10.1111/j.1467-9566.2005.00465.X

[8] Jingyun, T.; Guang, Y.; Xiaoxu, Y. A Comparative Study of Online Depression Communities in China. International Journal of Environmental Research and Public Health. 2020 17(14):5023 10.3390/ijerph17145023

[9] Santarossa S, Woodruff S J . \#SocialMedia: Exploring the Relationship of Social Networking Sites on Body Image, Self-Esteem, and Eating Disorders[J]. Social Media + Society,3,2(2017-5-01), 2017, 3(2):205630511770440.

[10] Holland, G.; Tiggemann, M., A systematic review of the impact of the use of social networking sites on body image and disordered eating outcomes. Body image 2016, 17, 100-110. 10.1016/j.bodyim.2016.02.008

[11] Saffran, K.; Fitzsimmons - Craft, E. E.; Kass, A. E.; Wilfley, D. E.; Taylor, C. B.; Trockel, M., Facebook usage among those who have received treatment for an eating disorder in a group setting. Int. J. Eating Disord. 2016, 49, 764-777. 10.1002/eat.22567

[12] Tiggemann M , Slater A . NetTweens: The Internet and Body Image Concerns in Preteenage Girls[J]. Journal of Early Adolescence, 2013, 34(5):606-620. 
[13] Fardouly J , Vartanian L R . Negative comparisons about one's appearance mediate the relationship between Facebook usage and body image concerns[J]. Body Image, 2015, 12(jan.):82-88.

[14] Sidani J E , Shensa A , Hoffman B , et al. The Association between Social Media Use and Eating Concerns among US Young Adults[J]. Journal of the Academy of Nutrition \& Dietetics, 2016:1465-1472.

[15] Branley D B , Covey J . Is exposure to online content depicting risky behavior related to viewers' own risky behavior offline?[J]. Computers in Human Behavior, 2017, 75:283-287.

[16] Branley D B , Judith C . Pro-ana versus Pro-recovery: A Content Analytic Comparison of Social Media Users' Communication about Eating Disorders on Twitter and Tumblr[J]. Frontiers in Psychology, 2017, 8:1356-.

[17] Arseniev-Koehler, A.; Lee, H.; McCormick, T.; Moreno, M. A., \# Proana: Pro-eating disorder socialization on twitter. J. Adolesc. Health 2016, 58, 659-664. 10.1016/j.jadohealth.2016.02.012

[18] Sowles S J , Mcleary M , Optican A , et al. A content analysis of an online pro-eating disorder community on Reddit[J]. Body Image, 2018, 24:137-144.

[19] Bert, F.; Gualano, M. R.; Camussi, E.; Siliquini, R., Risks and threats of social media websites: twitter and the proana movement. Cyberpsychology Behav. Soc. Netw. 2016, 19, 233-238. 10.1089/cyber.2015.0553

[20] Chancellor S , Lin Z J , Choudhury M D . "This Post Will Just Get Taken Down": Characterizing Removed Pro-Eating Disorder Social Media Content[C]// the 2016 CHI Conference. ACM, 2016.

[21] Taylor, C. B.; Bryson, S.; Luce, K. H.; Cunning, D.; Doyle, A. C.; Abascal, L. B.; Rockwell, R.; Dev, P.; Winzelberg, A. J.; Wilfley, D. E., Prevention of eating disorders in at-risk college-age women. Arch Gen Psychiat. 2006, 63, 881-888. 10.1001/archpsyc.63.8.881

[22] Zabinski, M. F.; Pung, M. A.; Wilfley, D. E.; Eppstein, D. L.; Winzelberg, A. J.; Celio, A.; Taylor, C. B., Reducing risk factors for eating disorders: Targeting at - risk women with a computerized psychoeducational program. Int. J. Eating Disord. 2001, 29, 401-408. 10.1002/eat.1036

[23] Forkner-Dunn, J., Internet-based patient self-care: the next generation of health care delivery. J. Med. Internet Res. 2003, 5. 10.2196/jmir.5.2.e8

[24] Ybarra, M. L.; Eaton, W. W., Internet-based mental health interventions. Mental health services research 2005, 7, 75-87. 10.1007/s11020-005-3779-8

[25] Gery W. Ryan, H. R. B., Techniques to identify themes. Field Methods 2003, 15, 85-109.

[26] Xianyun T , Philip B , Shuang S , et al. Characterizing Depression Issues on Sina Weibo[J]. International Journal of Environmental Research \& Public Health, 2018, 15(4):764.

[27] Yao X, Yu G, Tian X, et al. Patterns and Longitudinal Changes in Negative Emotions of People with Depression on Sina Weibo[J]. Telemedicine and e-Health, 2019, 26(6):734-743

[28] Siegel, S., Nonparametric statistics for the behavioral sciences. 1956.

[29] Hochreiter, S.; Schmidhuber, J., Long short-term memory. Neural Comput 1997, 9, 1735-1780. 10.1162/neco.1997.9.8.1735

[30] Tong, J.; Miao, S.; Wang, J.; Yang, F.; Lai, H.; Zhang, C.; Zhang, Y.; Hsu, G., A two-stage epidemiologic study on prevalence of eating disorders in female university students in Wuhan, China. Soc. Psychiatry Psychiatr. Epidemiol. 2014, 49, 499. 10.1007/s00127-013-0694-y

[31] Lee, S.; Lee, A. M., Disordered eating in three communities of China: A comparative study of female high school students in Hong Kong, Shenzhen, and rural Hunan. Int. J. Eating Disord. 2000, 27, 317-327. 10.1002/(SICI)1098-108X(200004)27:3<317::AID-EAT9>3.3.CO;2-U 